\title{
Spin-orbit alignment and magnetic activity in the young planetary system AU Mic ${ }^{\star}$
}

E. Martioli ${ }^{1,2}$, G. Hébrard ${ }^{1,3}$, C. Moutou ${ }^{4}$, J.-F. Donati ${ }^{4}$, É. Artigau $^{5}$, B. Cale ${ }^{6}$, N. J. Cook ${ }^{5}$, S. Dalal ${ }^{1}$, X. Delfosse ${ }^{7}$, T. Forveille ${ }^{7}$, E. Gaidos ${ }^{9}$, P. Plavchan ${ }^{6}$, J. Berberian ${ }^{6}$, A. Carmona ${ }^{7}$, R. Cloutier ${ }^{13}$, R. Doyon ${ }^{5}$, P. Fouqué ${ }^{8,4}$, B. Klein ${ }^{4}$, A. Lecavelier des Etangs ${ }^{1}$, N. Manset ${ }^{8}$, J. Morin ${ }^{10}$, A. Tanner ${ }^{11}$, J. Teske ${ }^{12}$, and S. Wang ${ }^{12}$

1 Institut d'Astrophysique de Paris, UMR7095 CNRS, Université Pierre \& Marie Curie, 98 bis Boulevard Arago, 75014 Paris, France

e-mail: martioli@iap.fr

2 Laboratório Nacional de Astrofísica, Rua Estados Unidos 154, Itajubá, MG 37504-364, Brazil

3 Observatoire de Haute Provence, St Michel l'Observatoire, France

${ }^{4}$ Univ. de Toulouse, CNRS, IRAP, 14 Avenue Belin, 31400 Toulouse, France

5 Université de Montréal, Département de Physique, IREX, Montréal, QC H3C 3J7, Canada

${ }^{6}$ George Mason University, 4400 University Drive, Fairfax, VA 22030, USA

7 Univ. Grenoble Alpes, CNRS, IPAG, 38000 Grenoble, France

8 Canada-France-Hawaii Telescope, CNRS, Kamuela, HI 96743, USA

9 Department of Earth Sciences, University of Hawaii at Mänoa, Honolulu, HI 96822, USA

10 Université de Montpellier, CNRS, LUPM, 34095 Montpellier, France

11 Mississippi State University, Department of Physics \& Astronomy, Hilbun Hall, Starkville, MS 39762, USA

12 Observatories of the Carnegie Institution for Science, 813 Santa Barbara Street, Pasadena, CA 91101, USA

13 Center for Astrophysics | Harvard \& Smithsonian, 60 Garden Street, Cambridge, MA 02138, USA

Received 18 June 2020 / Accepted 7 August 2020

\begin{abstract}
We present high-resolution near-infrared spectropolarimetric observations using the SPIRou instrument at Canada-France-Hawaii Telescope (CFHT) during a transit of the recently detected young planet AU Mic b, with supporting spectroscopic data from iSHELL at NASA InfraRed Telescope Facility. We detect Zeeman signatures in the Stokes $V$ profiles and measure a mean longitudinal magnetic field of $\bar{B}_{\ell}=46.3 \pm 0.7 \mathrm{G}$. Rotationally modulated magnetic spots likely cause long-term variations of the field with a slope of $\mathrm{d} B_{\ell} / \mathrm{d} t=-108.7 \pm 7.7 \mathrm{G} \mathrm{d}^{-1}$. We apply the cross-correlation technique to measure line profiles and obtain radial velocities through CCF template matching. We find an empirical linear relationship between radial velocity and $B_{\ell}$, which allows us to estimate the radial-velocity induced by stellar activity through rotational modulation of spots for the five hours of continuous monitoring of AU Mic with SPIRou. We model the corrected radial velocities for the classical Rossiter-McLaughlin effect, using MCMC to sample the posterior distribution of the model parameters. This analysis shows that the orbit of AU Mic b is prograde and aligned with the stellar rotation axis with a sky-projected spin-orbit obliquity of $\lambda=0_{-15^{\circ}}^{\circ}$. The aligned orbit of AU Mic b indicates that it formed in the protoplanetary disk that evolved into the current debris disk around AU Mic.
\end{abstract}

Key words. planetary systems - stars: individual: AU Mic - stars: activity - stars: magnetic field - techniques: radial velocities

\section{Introduction}

Detecting and characterizing planets around young stars is key to understanding the early stages of planetary evolution. Several mechanisms can produce strong misalignments between the planetary orbit and the stellar spin, including high-eccentricity tidal migration, planet-planet scattering, and Kozai-Lidov cycles driven by a binary (e.g., Dawson \& Johnson 2018; Triaud 2018). The resulting relative orientation of the planetary orbit and the

\footnotetext{
* Based on observations obtained at the Canada-France-Hawaii Telescope (CFHT) which is operated from the summit of Maunakea by the National Research Council of Canada, the Institut National des Sciences de l'Univers of the Centre National de la Recherche Scientifique of France, and the University of Hawaii. Based on observations obtained with SPIRou, an international project led by Institut de Recherche en Astrophysique et Planétologie, Toulouse, France.
}

rotation axis of the host star is a key discriminant between different formation and migration scenarios.

Here we report a measurement of the spin-orbit angle for the recently detected transiting super-Neptune planet AU Mic b (Plavchan et al. 2020). AU Mic is a young and active M1 star with a spatially resolved edge-on debris disk (Kalas et al. 2004), and is a member of the $\beta$ Pictoris Moving Group (Torres et al. 2006). Its distance of only $9.7248 \pm 0.0046 \mathrm{pc}$ (Gaia Collaboration 2018) and its estimated age of $22 \pm 3 \mathrm{Myr}$ (Mamajek \& Bell 2014) make it both the closest and the youngest system with either a spatially resolved edge-on debris disk or a transiting planet. Table 1 summarizes the stellar and planetary parameters of the system.

Young systems with detected planets (e.g., V830 Tau b; Donati et al. 2016), and especially those with either a remnant debris disk like $\beta$ Pic b (Lagrange et al. 2009) or transiting 
Table 1. Star and planet $b$ parameters for AU Mic system.

\begin{tabular}{lcc}
\hline \hline Parameter & Value & Ref. \\
\hline$T_{\text {eff }}$ & $3700 \pm 100 \mathrm{~K}$ & 1 \\
Star mass & $0.50 \pm 0.03 M_{\odot}$ & 1 \\
Star radius & $0.75 \pm 0.03 R_{\odot}$ & 2 \\
$P_{\text {rot }}$ & $4.863 \pm 0.010$ days & 1 \\
$v_{\mathrm{e}}=2 \pi R_{\star} / P_{\text {rot }}$ & $7.8 \pm 0.3 \mathrm{~km} \mathrm{~s}^{-1}$ & 2,1 \\
Age & $22 \pm 3 \mathrm{Myr}$ & 3 \\
Distance & $9.7248 \pm 0.0046 \mathrm{parsec}$ & 4 \\
Limb dark. coef. $\mu_{H}$ & 0.3016 & 5 \\
\hline Time of conjunction & $2458330.39153_{-0.00068}^{+0.00070} \mathrm{BJD}$ & 1 \\
Transit duration & $3.50_{-0.59}^{+0.63} \mathrm{~h}$ & 1 \\
Orbital period & $8.46321 \pm 0.00004$ days & 1 \\
RV semi-amplitude & $<28 \mathrm{~m} \mathrm{~s}^{-1}$ & 1 \\
$R_{\mathrm{p}} / R_{\star}$ & $0.0514 \pm 0.0013$ & 1 \\
$a_{\mathrm{p}} / R_{\star}$ & $19.1_{-1.6}^{+1.8}$ & 1 \\
Impact parameter $(b)$ & $0.16_{-0.14}^{+0.14}$ & 1 \\
Orbit inclination $\left(i_{\mathrm{p}}\right)$ & $89.5^{\circ} \pm 0.4^{\circ}$ & 6 \\
\hline
\end{tabular}

References. (1) Plavchan et al. (2020); (2) White et al. (2015); (3) Mamajek \& Bell (2014); (4) Gaia Collaboration (2018); (5) Claret \& Bloemen (2011); (6) $\cos i_{\mathrm{p}}=\frac{b}{a_{\mathrm{p}} / R_{\star}}$.

planets (e.g., K2-33 b, DS Tuc Ab; David et al. 2016; Mann et al. 2016; Newton et al. 2019) are key probes of planetary formation. AU Mic has both a disk and at least one transiting planet, and is also unique among debris disk hosts for being an $\mathrm{M}$ star, the most numerous type of star in our Galaxy and the most promising spectral type to find habitable planets using current techniques.

\section{Observations and data reduction}

\subsection{SPIRou}

The Spectro-Polarimètre Infra Rouge $(\mathrm{SPIRou})^{1}$ is a stabilized high-resolution near-infrared (NIR) spectro-polarimeter (Donati et al. 2020) mounted on the $3.6 \mathrm{~m}$ Canada-FranceHawaii Telescope (CFHT) atop Maunakea, Hawaii. SPIRou is designed to perform high-precision measurements of stellar radial velocities to search for and characterize exoplanets. It provides full coverage of the NIR spectrum from $950 \mathrm{~nm}$ to $2500 \mathrm{~nm}$ in a single exposure without gaps, and at a spectral resolving power of $\lambda / \Delta \lambda \sim 70000$. Its high throughput in the NIR makes SPIRou an ideal instrument to follow up transiting exoplanets around cool stars. SPIRou allows simultaneous spectropolarimetry, which helps identify stellar magnetic activity and is especially important for active late-type stars (Morin et al. 2010) and young stellar objects. AU Mic is both cool and young, with high magnetic activity (Berdyugina et al. 2008; Afram \& Berdyugina 2019), which means that polarimetric information is required in order to make a reliably diagnosis.

\subsection{Observations}

We observed the June 16, 2019 transit of AU Mic b as part of the Work Package 2 (WP2) of the SPIRou Legacy Survey (Donati et al. 2020) CFHT large program (id 19AP42, PI: Jean-François Donati). The observations were carried out in the Stokes $V$ spectropolarimetric mode of SPIRou. They

\footnotetext{
1 More information about SPIRou in http://spirou.irap.omp.eu and https://www.cfht.hawaii.edu/Instruments/SPIRou/
}

started at UT 2019-06-17T10:10:56 and finished at UT 201906-17T15:13:45, and consist of 116 individual flux spectra of AU Mic with a $122.6 \mathrm{~s}$ exposure time. These correspond to 29 Stokes $V$ polarimetric spectra (with $4 \times$ individual exposures per polarimetry sequence). Our observations started with an air mass of 2.9 and ended at 1.8 , with a minimum of 1.59 . The conditions remained nearly photometric, with the SkyProbe monitor (Cuillandre et al. 2004) measuring a maximum absorption of $0.12 \mathrm{mag}$. The image quality (seeing) measured by the SPIRou guider varies from 0.8 to $1.6 \mathrm{arcsec}$, with a mean value of $0.96 \pm 0.13$ arcsec. The Moon was almost full, with $99 \%$ illumination, and was separated from our target by $40.3^{\circ}$. The peak signal-to-noise ratio $(\mathrm{S} / \mathrm{N})$ per spectral bin (in the spectral order centered at $\sim 1670 \mathrm{~nm}$ ) of the individual exposures varies between 176 and 273, with a mean value of 242 .

\subsection{Data reduction}

The data were reduced with version v.0.6.082 of the APERO SPIRou data reduction software (Cook et al., in prep.). APERO first combines the sub-exposures at the read-out level, correcting for nonlinearity in the pixel-by-pixel response. The 1D spectral fluxes are optimally extracted following Horne (1986). The individual spectral orders are processed and saved separately, providing a 2D frame with about 4088 spectral pixels for 48 orders. SPIRou uses two optical fibers to collect light from the two images formed by a Wollaston prism. For pure spectroscopy, APERO merges the spectra of the two beams, whereas for polarimetry the fluxes of the two channels are individually saved for later polarimetric analysis. APERO also calculates a blaze function for each channel from daytime flat-field exposures. The pixel-to-wavelength calibration is obtained from a combination of daytime exposures of a Uranium-Neon hollowcathode lamp and of a thermally controlled Fabry-Pérot etalon (FP). The FP also feeds a third fiber during science exposures to monitor instrument drifts. APERO calculates a telluric absorption spectrum for each exposure using an extensive library of telluric standard stars observed nightly with SPIRou over a wide range of air mass and atmospheric conditions. APERO uses the PCA-based correction technique of Artigau et al. (2014) to produce a telluric-absorption corrected spectrum. APERO also calculates the cross-correlation function (CCF) with a set of line masks optimized for different stellar types and systemic velocities.

\section{4. iSHELL data}

We include in our analysis simultaneous radial velocity (RV) measurements from 47 in-transit spectra of the June 16, 2019 transit of $\mathrm{AU}$ Mic obtained with the iSHELL spectrometer $(\lambda / \Delta \lambda \sim 80000)$ on the NASA InfraRed Telescope Facility (IRTF, Rayner et al. 2016). AU Mic was observed in KGAS mode $(2.1-2.5 \mu \mathrm{m})$ from UT 2019-06-17T11:08:19 to UT 201906-17T12:53:32. Their two-minute exposure time results in a photon $\mathrm{S} / \mathrm{N}$ of $\sim 60-70$ per spectral pixel at $2.4 \mu \mathrm{m}$ (the approximate peak of the blaze function for the center order), and in turn in a RV precision of $15-27 \mathrm{~m} \mathrm{~s}^{-1}$ (median $21 \mathrm{~m} \mathrm{~s}^{-1}$ ) per measurement. These spectra were reduced and their RVs extracted using the methods outlined in Cale et al. (2019). The RV data measured by iSHELL are presented in Appendix A.

\section{Spectropolarimetry}

SPIRou Stokes- $V$ spectra are obtained from sequences of four exposures with distinct positions of the Fresnel rhombs such 


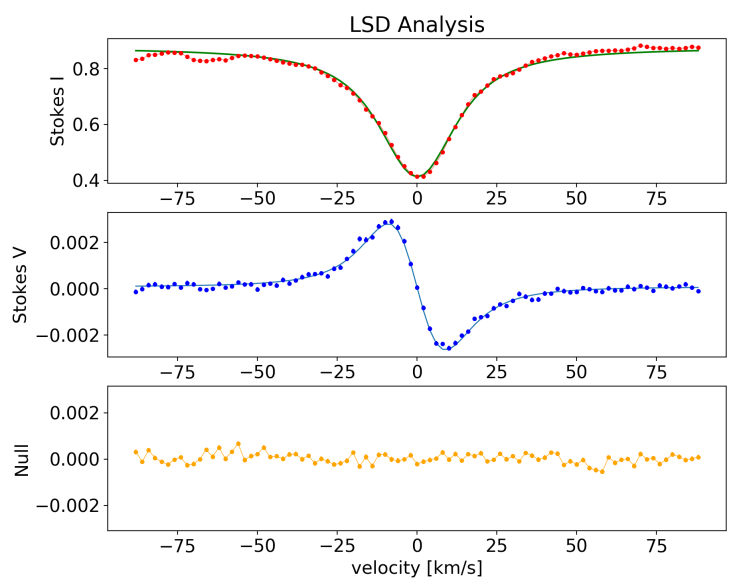

Fig. 1. Median of all LSD profiles in the AU Mic time series. Top panel: Stokes I LSD (red points) with a a Voigt profile model fit (green line); middle panel: Stokes $V$ (blue points) and a double Voigt profile model fit (blue line); bottom panel: null polarization profile (orange points).

that systematic errors affecting the polarimetric analysis are minimized (we compute the Stokes parameter using the "ratio" method Donati et al. 1997; Bagnulo et al. 2009). Since the order of the exposures within the successive AU Mic polarimetric sequences is identical and the angles of the retarder within each sequence are set to alternate positions, one can obtain higher time sampling by calculating polarimetric spectra in every set of four adjacent exposures. With this method we obtain a total of 113 (non-independent) polarimetric spectra of AU Mic, instead of the 29 that would be obtained from analyzing each sequence separately.

We applied the least squares deconvolution (LSD) method of Donati et al. (1997) to each Stokes $I$, Stokes $V$, and null polarization spectrum to obtain LSD profiles for each. The line mask used in our LSD analysis was obtained from the VALD catalog (Piskunov et al. 1995) based on a MARCS model atmosphere (Gustafsson et al. 2008) with an effective temperature of $3500 \mathrm{~K}$ and surface gravity of $\log g=5.0 \mathrm{~cm} \mathrm{~s}^{-2}$. A total of 1363 lines were included in the LSD analysis.

Figure 1 presents the medians of the 113 profiles, and its Stokes- $V$ panel shows a clearly detected Zeeman signature. We fit a Voigt function to the Stokes-I profile and a double Voigt function to the Stokes- $V$ profile, both presented in Fig. 1. The Voigt model is a good approximation for the profiles of AU Mic, which confirms a significant contribution from Lorentzian broadening mechanisms, most likely due to its high surface gravity. A complete analysis of the line profiles considering the several broadening mechanisms in AU Mic is out of the scope of this paper. The fit profiles are also important in this work to correct for the velocity shift in the profiles, which is needed for the calculation of the longitudinal magnetic field as given by Eq. (1) in Sect. 6.

\section{Radial velocities}

We measure the radial velocity of $\mathrm{AU}$ Mic using the $\mathrm{CCF}$ between the telluric-corrected stellar spectrum and a line mask (Pepe et al. 2002). The broad NIR band pass of SPIRou covers thousands of atomic and molecular lines, which greatly improves the precision in the determination of the CCF. The line mask plays an important role in the CCF method, since it determines the spectral regions that are probed and the statistical weight for each of these regions. We use the "M2_weighted_RV_-5.mas" line mask from the set of empirical masks delivered by the APERO pipeline. This mask is based on the observed spectra of the M2V star Gl 15A and is a good match to the M1V spectral type of AU Mic.

Even though SPIRou spectra are corrected for telluric absorption, this correction can create additional noise in the spectrum. This noise was evaluated using SPIRou commissioning data, and was taken into account in the weight of each line in the mask, which is decreased by a factor proportional to the extra noise. The lines are blanked out if they are impacted by telluric deeper than $40 \%$ absorption at a systemic radial velocity of $-5 \mathrm{~km} \mathrm{~s}^{-1}$ (which is close to the $4.5 \mathrm{~km} \mathrm{~s}^{-1}$ systemic velocity of $\mathrm{AU}$ Mic) with a window of $\pm 33 \mathrm{~km} \mathrm{~s}^{-1}$ (maximum of barycentric velocity). For a telluric absorption of $10 \%, 20 \%, 30 \%$, and $40 \%$, the weights are given by the line depth divided by a factor of $1.5,3,7$, and 16 , respectively.

The mask has 3475 lines, but we further filter it using the approach of Moutou et al. (2020), eliminating those lines which are not detected in the mean Stokes- $I$ spectrum of AU Mic, for a final set of 2277 retained lines. We note that one could have obtained the radial velocities from the LSD Stokes- $I$ profiles as presented in Sect. 3. However, our LSD analysis is restricted to spectral lines with a known Landé factor, which is smaller compared to the number of lines in the CCF analysis, resulting in larger uncertainties in radial velocities.

The 48 orders delivered by SPIRou have different noise levels, depending mostly on the instrumental throughput (Donati et al. 2020) and on the telluric absorption. We compute a separate $\mathrm{CCF}$ for each spectral order, and combine some of those into a sum CCF to improve precision. We obtain individual RV measurements for each spectral order and calculate the $\mathrm{RV}$ dispersion, $\sigma_{\mathrm{RV}}$, given by the standard deviation throughout the time series. The mean RV dispersion between all orders is $\bar{\sigma}_{\mathrm{RV}}=97 \pm 90 \mathrm{~m} \mathrm{~s}^{-1}$. Given the variable RV precision between orders, we decided to restrict our analysis to the seven orders in the $1512 \mathrm{~nm}-1772 \mathrm{~nm}$ range in the $\mathrm{H}$-band, where the mean $\mathrm{RV}$ dispersion is $\bar{\sigma}_{\mathrm{RV}}=28 \pm 7 \mathrm{~m} \mathrm{~s}^{-1}$. Our CCF mask has a total of 842 lines within this spectral range.

We measure radial velocities from the CCF by least-square fitting for the velocity shift $\Delta v_{i}$ that best matches the CCF of an individual exposure, $\mathrm{CCF}_{i}$, to the median of the CCFs of all exposures, $\mathrm{CCF}_{m}$. The shifted template $\mathrm{CCF}_{m}(v+\Delta v)$ is calculated by cubic interpolation. We also measured RVs by fitting a Gaussian to each $\mathrm{CCF}_{i}$, which is the most commonly used method. This gives similar results but shows stronger systematic error correlated with the air mass of the observations, and we therefore adopt the CCF matching (CM) method in our analysis. In yet another processing alternative, we apply a median filter (MF) to the CCF time series before calculating RVs through template matching using a $3 \times 3$ window along the time and velocity axes. The RV data measured by SPIRou are presented in Appendix A.

\section{Rossiter-McLaughlin effect}

We first model the SPIRou radial velocities of AU Mic obtained from the median-filtered CCFs, as the combination of its reflex orbital motion caused by planet $b$, assuming a circular orbit and the Plavchan et al. (2020) orbital parameters, and the classical Rossiter-McLaughlin (RM) effect, with the stellar limb darkening accounted for as described in Ohta et al. (2005). We adopt a linear limb-darkening model and fix the $H$-band coefficient to $\mu_{H}=0.3016$ from Claret \& Bloemen (2011). 
Table 2. Fit parameters of AU Mic b.

\begin{tabular}{lcc}
\hline \hline Parameter & Prior & Posterior \\
\hline$T_{\mathrm{c}}$ & $\mathcal{N}(0.39153,0.00070)$ & $0.3892 \pm 0.0007$ \\
$R_{\mathrm{p}} / R_{\star}$ & $\mathcal{N}(0.0514,0.0013)$ & $0.063 \pm 0.004$ \\
$\lambda$ & $\mathcal{U}(-180,180)$ & $-0.2_{-19.3}^{+18.9}$ \\
$\nu_{\mathrm{e}} \sin i_{\star}$ & $\mathcal{N}(7.8,0.3)$ & $7.5 \pm 0.9$ \\
$\gamma$ & $\mathcal{U}(-\infty, \infty)$ & $-4.3869 \pm 0.0005$ \\
$\alpha$ & $\mathcal{U}(-\infty, \infty)$ & $149 \pm 9$ \\
\hline
\end{tabular}

Notes. $T_{\mathrm{c}}$ is in units of BJD $-2458330, \lambda$ is in degrees, $v_{\mathrm{e}} \sin i_{\star}$ and $\gamma$ are in $\mathrm{km} \mathrm{s}^{-1}$, and $\alpha$ is in $\mathrm{m} \mathrm{s}^{-1} \mathrm{~d}^{-1}$. The symbol $\mathcal{N}(\mu, \sigma)$ represents a normal distribution with mean $\mu$ and standard deviation $\sigma$, and $\mathcal{U}\left(x_{1}, x_{2}\right)$ represents a uniform distribution with minimum and maximum values given by $x_{1}$ and $x_{2}$.

We adopt as free parameters the time of conjunction $T_{\mathrm{c}}$, the planet-to-star-radius ratio $R_{\mathrm{p}} / R_{\star}$, the sky-projected obliquity angle $\lambda$, the projected stellar rotation velocity $v_{\mathrm{e}} \sin i_{\star}$, the systemic velocity $\gamma$, and we include a slope of the RVs as a function of time, $\alpha$, to account for both stellar activity trends and a planetary signal. Table 2 shows the priors which we adopt for each parameter. We sampled the posterior distributions using the emcee Markov chain Monte Carlo (MCMC) package (Foreman-Mackey et al. 2013), using 50 walkers and 2000 MCMC steps of which we discard the first 500. The best-fit values in Table 2 are the medians of the posterior distribution, and the error bars enclose $34 \%$ on each side of the median. The MCMC samples and posterior distributions are illustrated in Fig. C.1.

Figure 2 shows as blue triangles the SPIRou AU Mic RVs obtained by CCF matching the original CCFs, whereas the filled circles show those obtained from the median filtered CCFs. We identify two anomalous regions in the time series, marked in red in the figure, where the RV residuals are above $2.5 \times \sigma$. We interpret these regions as stellar activity events, such as spot-crossing by the planet and/or flares. The corresponding data were masked out in the final model fit. Our best-fit RM model includes a RV slope of $149 \pm 9 \mathrm{~m} \mathrm{~s}^{-1} \mathrm{~d}^{-1}$ and the dispersion of its residuals is $5.1 \mathrm{~m} \mathrm{~s}^{-1}$ for data that were not masked out. For illustration of the stability of SPIRou, we also show the instrumental drifts obtained from the spectrum of the FP calibrator which is simultaneously observed through the reference fiber, with a dispersion of just $0.51 \mathrm{~m} \mathrm{~s}^{-1}$.

The sky-projected obliquity angle of $\lambda=-0.2_{-19.3}^{+18.9}$ degrees shows that the orbit of AU Mic b is prograde and close to aligned with the rotation axis of the parent star. Our best-fit value of $v_{\mathrm{e}} \sin i_{\star}=7.5 \pm 0.9 \mathrm{~km} \mathrm{~s}^{-1}$ agrees at a level of $2 \times \sigma$ with independent measurements of $v_{\mathrm{e}} \sin i_{\star}=8.7 \pm 0.2 \mathrm{~km} \mathrm{~s}^{-1}$ (Gaidos et al. 2014). Our analysis also shows that the conjunction occurred about $3.4 \mathrm{~min}\left(\sim 3 \times \sigma_{T_{\mathrm{c}}}\right)$ earlier than predicted, and favors a slightly larger planetary radius, though within $3 \sigma_{R_{\mathrm{p}} / R_{\star}}$.

\section{Magnetic activity}

As amply illustrated by its TESS light curve, AU Mic is an active star, with a surface largely filled by spots, and with frequent flares (Plavchan et al. 2020). The $\sim 5 \mathrm{~h}$ SPIRou time series covers $4.3 \%$ of the 4.863 -day rotation period of AU Mic. The nonuniform brightness distribution of the AU Mic disk has therefore probably changed slowly through rotation of the visible hemisphere, and rapidly through flaring and spot evolution. Planet AU Mic b can additionally transit spots, also causing fast variability. These brightness variations change the rotation profile of AU Mic and strongly affect our RV measurements.

Since both spot and flare events are connected to the magnetic field (Lavail et al. 2018), we search for an empirical correlation between the measured RVs and the longitudinal magnetic field $B_{\ell}$, in an attempt to mitigate the effects of stellar activity on our RV data. The longitudinal magnetic field $B_{\ell}$ is calculated for each AU Mic polarized spectrum using the following equation from Donati et al. (1997):

$B_{\ell}=-2.14 \times 10^{11} \frac{\int v V(v) \mathrm{d} v}{\lambda_{0} \cdot g_{\mathrm{eff}} \cdot c \cdot \int\left[I_{\mathrm{c}}-I(v)\right] \mathrm{d} v}$,

where $c$ is the speed of light, $I(v)$ and $V(v)$ are the Stokes $I$ and $V$ profiles as functions of velocity $v$ in the frame of the star, $I_{\mathrm{c}}$ is the continuum of the Stokes $I$ profile, $\lambda_{0}=1515.38 \mathrm{~nm}$ is the mean wavelength, and $g_{\mathrm{eff}}=1.24$ is the mean Landé factor of the lines included in the mask. The $B_{\ell}$ data are provided in Appendix B. The bottom panel of Fig. 3 illustrates our measurements of $B_{\ell}$ for AU Mic, showing values obtained both from the original Stokes $V$ profiles (black points with error bars) and from the median filtered profiles (black line). The mean longitudinal field of AU Mic during our time series is $\bar{B}_{\ell}=47.9 \pm 8.1 \mathrm{G}$, with a linear trend of slope $-108.7 \pm 7.7 \mathrm{G} \mathrm{d}^{-1}$ which is likely due to rotational modulation of spots. The field measured from the median LSD profile of Fig. 1 is $\bar{B}_{\ell}=46.3 \pm 0.7 \mathrm{G}$ and therefore closely matches the mean longitudinal field of the sequence.

We least-square fit (Fig. 3, top panel) the following linear function to the RM-subtracted RV data:

$v_{B}(t)=\left[B_{\ell}(t)-B_{0}\right] a+v_{0}$,

where $B_{0}$ is an arbitrary reference magnetic field, $a$ is the scaling factor between the two quantities, and $v_{0}$ is a constant velocity. The best-fit scaling factor is $a=-1.34 \pm 0.12 \mathrm{~m} \mathrm{~s}^{-1} \mathrm{G}^{-1}$, significant at the $11 \sigma$ level. The Pearson- $r$ correlation coefficient between our measured RVs and the predicted $v_{B}$ is $r=0.72$ with a $p$-value of $3.7 \times 10^{-19}$, showing a significant correlation between the two quantities, mainly because stellar rotation modulates both the RVs and $B_{\ell}$. Subtracting a linear fit from both $B_{\ell}$ and $\mathrm{RVs}$ to eliminate the long-term variations reduces $r$ to 0.19 with a $p$-value of $4.6 \times 10^{-2}$, showing some possible smaller correlation between the short-timescale variations of the RVs and $B_{\ell}$. However, subtracting only the fitted $B_{\ell}$ slope produces less dispersed RV residuals than subtracting the full empirical model $v_{B}$. The short-timescale structure is likely due to spot evolution, flares, and the planet transiting spots. Each of these phenomena unfortunately has a different relationship between its RV variation and $B_{\ell}$, which makes our linear model much too simple to account for the short-term RV variability. A future paper will investigate these issues in much more detail and with an extended observational data set.

\section{Results and discussion}

Our preferred SPIRou RVs of AU Mic are obtained by subtracting from the measured RVs the linear component of the empirical model, a $145 \pm 17 \mathrm{~m} \mathrm{~s}^{-1} \mathrm{~d}^{-1}$ slope which mostly removes the stellar activity signal discussed above. We then adjust the RM model of Sect. 5 to both the iSHELL and corrected SPIRou data using 50 MCMC walkers and 2000 steps with the first 500 discarded. We consider two systemic velocities, $\gamma_{\text {SPIRou }}$ and $\gamma_{\text {iSHELL }}$, to account for different instrumental zero points. The MCMC samples and posterior distributions are illustrated in Fig. C.2. 


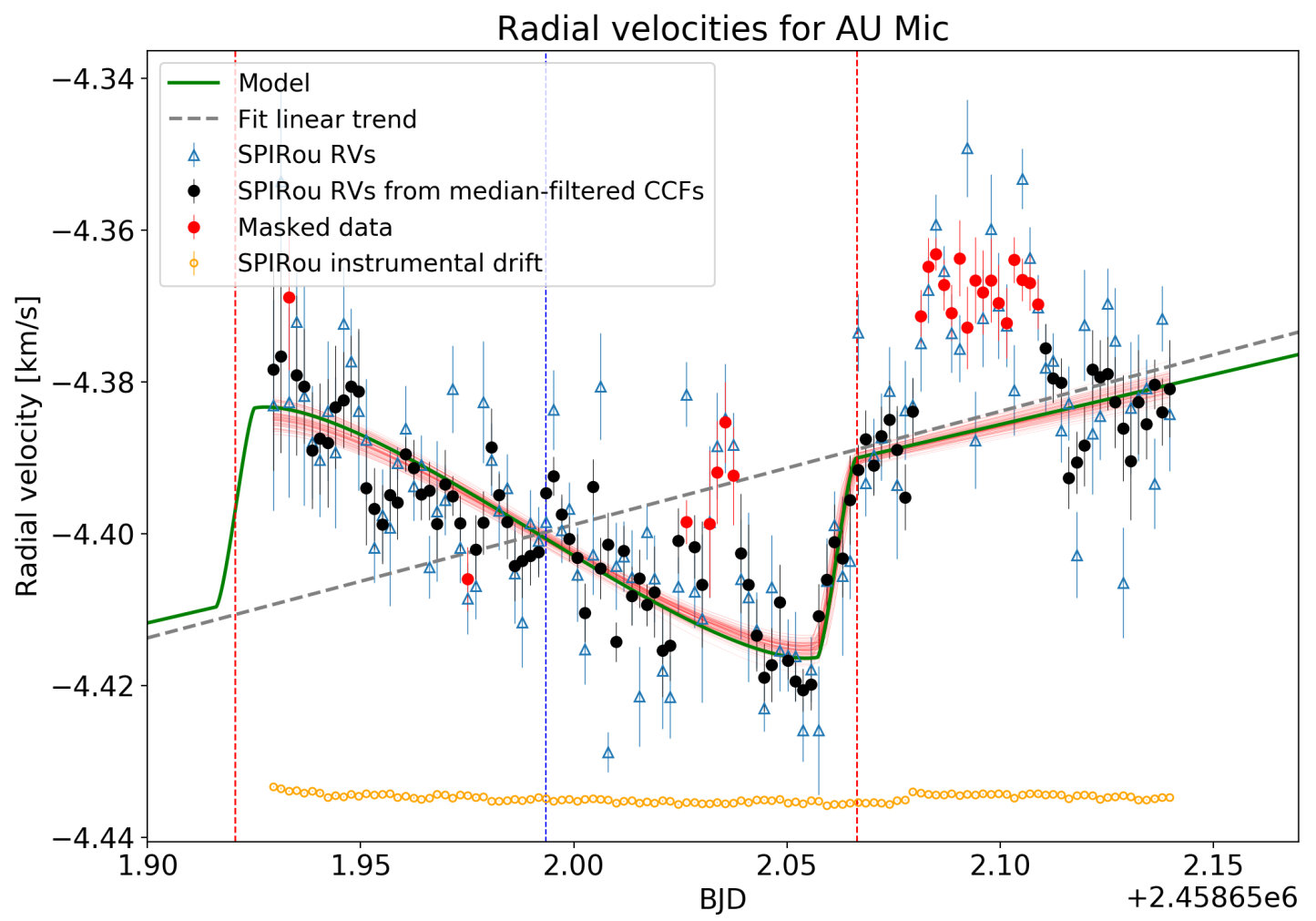

Fig. 2. SPIRou radial velocities of AU Mic. Blue triangles show the RVs obtained from CCF matching the original CCFs, and filled circles show the RVs obtained from CCF matching the median-filtered CCFs. Red circles show data masked by our $2.5 \sigma$ clip. Vertical lines show the predicted start, center, and end of the transit. The green line shows the best-fit model and the thin red lines show models for 100 randomly selected MCMC samples. The gray dashed line shows best-fit slope of $149 \pm 9 \mathrm{~m} \mathrm{~s}^{-1} \mathrm{~d}^{-1}$ with an arbitrary vertical offset for visualization, and the orange points show the SPIRou instrumental drift (also with an arbitrary offset), and illustrate its dispersion of just $0.51 \mathrm{~m} \mathrm{~s}^{-1}$.

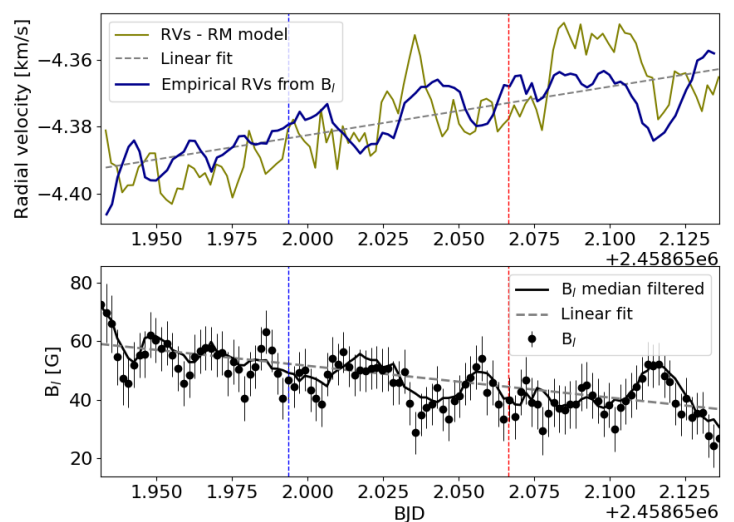

Fig. 3. Top panel: RM-subtracted RVs of AU Mic as a function of time (green) and their best-fit Eq. (2) linear model (dark blue). The vertical dashed lines show the predicted transit center (blue) and end (red). Bottom panel: longitudinal field derived from the original LSD profiles (black circles) and from the median filtered LSD profiles (black line). We also present a linear fit to the values of $B_{\ell}$ (dashed grey line in bottom panel) and the corresponding trend in velocity space (dashed gray line in top panel).

The final fit parameters are presented in Table 3. We obtain a fitted obliquity angle of $\lambda=-0.3_{-15.0^{\circ}}^{\circ+17.8^{\circ}}$ and $5.1 \mathrm{~m} \mathrm{~s}^{-1}$ and $11.5 \mathrm{~m} \mathrm{~s}^{-1}$ dispersions for the SPIRou (masked data excluded) and iSHELL residuals. This result confirms that the planet is on a prograde orbit and that the orbital and rotation spins are closely aligned. Figure 4 shows this final fit model to the RV data for both instruments.
Table 3. Final fit parameters for the Rossiter-McLaughlin model of AU Mic b using both SPIRou and iSHELL data sets.

\begin{tabular}{lcc}
\hline \hline Parameter & Prior & Posterior \\
\hline$T_{\mathrm{c}}$ & $\mathcal{N}(0.39153,0.00070)$ & $0.3897 \pm 0.0006$ \\
$R_{\mathrm{p}} / R_{\star}$ & $\mathcal{N}(0.0514,0.0013)$ & $0.061 \pm 0.002$ \\
$\lambda$ & $\mathcal{U}(-180,180)$ & $-0.3_{-15.0}^{+17.8}$ \\
$v_{\mathrm{e}} \sin i_{\star}$ & $\mathcal{N}(7.8,0.3)$ & $7.8 \pm 0.6$ \\
$\gamma_{\text {SPIRou }}$ & $\mathcal{U}(-\infty, \infty)$ & $-4.3808 \pm 0.0005$ \\
$\gamma_{\text {iSHELL }}$ & $\mathcal{U}(-\infty, \infty)$ & $0.027 \pm 0.003$ \\
\hline
\end{tabular}

Notes. $T_{\mathrm{c}}$ is in units of BJD $-2458330, \lambda$ is in degrees, $v_{\mathrm{e}} \sin i_{\star}, \gamma_{\mathrm{SPIRou}}$ and $\gamma_{\text {iSHELL }}$ are in $\mathrm{km} \mathrm{s}^{-1}$.

Since iSHELL only observed a partial transit of AU Mic and no out-of-transit baseline, its data alone do not constrain a full RM model independently of SPIRou, but the two data sets are fully compatible. The agreement between the data sets from these two different instruments using independent techniques for data analysis is remarkable and shows that both instruments are stable and can provide RVs with precisions of a few $\mathrm{m} \mathrm{s}^{-1}$ for an active star.

In addition to the analysis presented here, we performed extensive tests adopting different model assumptions, and obtaining radial velocities with different methods including RV measurements from an analysis of CCF bisector and measuring RVs from LSD profiles produced by an independent pipeline (Donati et al.2020). All RM model fits persistently prefer a $\lambda$ value consistent with aligned rotation and orbital angular momenta. 


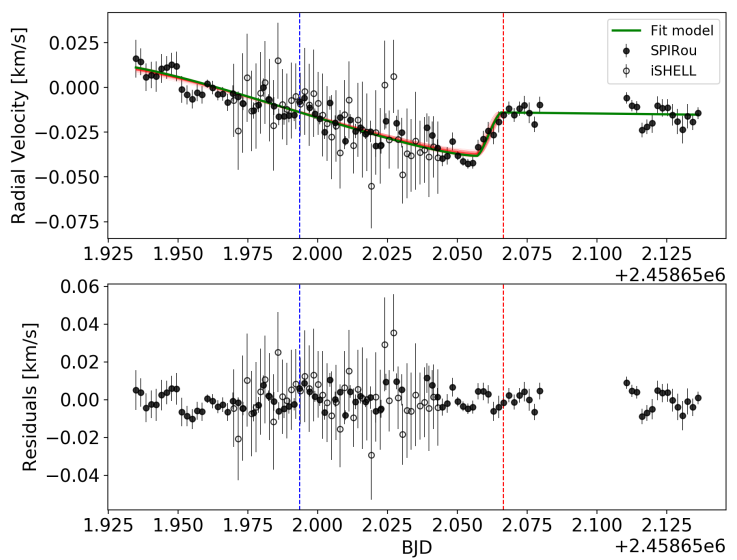

Fig. 4. Simultaneous fit to the corrected SPIRou RVs (filled circles) and iSHELL RVs (hollow circles) of the model of Rossiter-McLaughlin effect (green line shows the best-fit model and the thin red lines show models for 100 randomly selected MCMC samples). The vertical dashed lines show the predicted transit center (blue) and end (red) Bottom panel: residuals of the fit with respective dispersions of $5.1 \mathrm{~m} \mathrm{~s}^{-1}$ and $11.5 \mathrm{~m} \mathrm{~s}^{-1}$ for SPIRou and iSHELL.

\section{Conclusions}

We present observations of a transit of the recently detected planet of the nearby young M1 star AU Mic with a resolved edge-on debris disk with the SPIRou high-resolution NIR spectropolarimeter at CFHT and the iSHELL high-resolution NIR spectrograph at IRTF.

We cross-correlate the SPIRou spectra with numerical masks and employ the CCF matching method to obtain radial velocities of AU Mic with $\sim 5 \mathrm{~m} \mathrm{~s}^{-1}$ precision. We obtain Stokes $I$ and $V$ spectra of AU Mic and perform a LSD analysis to obtain average Stokes $I$ and $V$ profiles, and strongly detect a Zeeman signature in the Stokes $V$ profile. The corresponding mean longitudinal magnetic field is $\bar{B}_{\ell}=46.3 \pm 0.7 \mathrm{G}$ and varies at a global rate of $\mathrm{d} B_{\ell} / \mathrm{d} t=-108.7 \pm 7.7 \mathrm{G} \mathrm{d}^{-1}$. We use the correlated variability of the longitudinal magnetic field and radial velocity, with a scaling factor of $a=-1.34 \pm 0.12 \mathrm{~m} \mathrm{~s}^{-1} \mathrm{G}^{-1}$, to empirically correct a linear RV trend of $145 \pm 17 \mathrm{~m} \mathrm{~s}^{-1} \mathrm{~d}^{-1}$. This trend is consistent with the slope of $149 \pm 9 \mathrm{~m} \mathrm{~s}^{-1} \mathrm{~d}^{-1}$ found in our RM analysis and compatible with the expected levels of RV jitter of AU Mic in the NIR (Bailey et al. 2012).

We fit a classical Rossiter-McLaughlin effect model to the SPIRou and iSHELL data, and find a sky-projected spin-orbit obliquity angle for AU Mic b of $\lambda=0_{-15^{\circ}}^{\circ}$. AU Mic b is therefore on a prograde and closely aligned orbit, which is evidence that the planet likely formed in the protoplanetary disk that evolved into the current $\mathrm{AU}$ Mic debris disk, provided that the star-disk-planet components of the system share the same angular momentum orientation.

Acknowledgements. The authors wish to recognize and acknowledge the very significant cultural role and reverence that the summit of Maunakea has always had within the indigenous Hawaiian community. We are most fortunate to have the opportunity to conduct observations from this mountain. We acknowledge funding from the French National Research Agency (ANR) under contract num- ber ANR-18-CE31-0019 (SPlaSH) and also in the framework of the Investissements dAvenir program (ANR-15-IDEX-02), through the funding of the "Origin of Life" project of the Univ. Grenoble-Alpes. We also acknowledge funding from the European Research Council (ERC) under the H2020 research and innovation programme (grant agreement 740651 NewWorlds). SPIRou project is funded by the IDEX initiative at UFTMP, UPS, the DIMACAV programme in Region Ile de France, the MIDEX initiative at AMU, the Labex@OSUG2020 programme, UGA, INSU/CNRS, CFI, CFHT, LNA, CAUP and DIAS. We are also grateful for generous amounts of in-kind manpower allocated to SPIRou by OMP/IRAP, OHP/LAM, IPAG, CFHT, NRC-H, UdeM, UL, OG, LNA and ASIAA. P.P. acknowledges support from the NASA Exoplanet Exploration Program and the National Science Foundation (Astronomy and Astrophysics grant 1716202). J.M. acknowledges support from Eric Stempels and Nikolai Piskunov of the VALD team at Uppsala University. J.M. also thanks Benjamin Tessore (IPAG), Eric Josselin (LUPM) and Agnés Lébre (LUPM) for their contribution to the Montpellier VALD local mirror and Bertrand Plez (LUPM) for his assistance with MARCS model atmospheres.

\section{References}

Afram, N., \& Berdyugina, S. V. 2019, A\&A, 629, A83

Artigau, É., Astudillo-Defru, N., Delfosse, X., et al. 2014, in Society of PhotoOptical Instrumentation Engineers (SPIE) Conference Series, Proc. SPIE, 9149, 914905

Bagnulo, S., Landolfi, M., Landstreet, J. D., et al. 2009, PASP, 121, 993

Bailey, J. I., III, White, R. J., Blake, C. H., et al. 2012, ApJ, 749, 16

Berdyugina, S. V., Berdyugin, A. V., Fluri, D. M., \& Piirola, V. 2008, ApJ, 673, L83

Cale, B., Plavchan, P., LeBrun, D., et al. 2019, AJ, 158, 170

Claret, A., \& Bloemen, S. 2011, VizieR Online Data Catalog: J/A+A/529/A75

Cuillandre, J. C., Magnier, E. A., Isani, S., et al. 2004, in Scientific Detectors for Astronomy, eds. P. Amico, J. W. Beletic, \& J. E. Beletic (Dordrecht: Springer Netherlands), 287

David, T. J., Hillenbrand, L. A., Petigura, E. A., et al. 2016, Nature, 534, 658

Dawson, R. I., \& Johnson, J. A. 2018, ARA\&A, 56, 175

Donati, J. F., Semel, M., Carter, B. D., Rees, D. E., \& Collier Cameron, A. 1997, MNRAS, 291, 658

Donati, J. F., Moutou, C., Malo, L., et al. 2016, Nature, 534, 662

Donati, J. F., Kouach, D., Moutou, C., et al. 2020, MNRAS, submitted [arXiv:2008.08949]

Foreman-Mackey, D., Hogg, D. W., Lang, D., \& Goodman, J. 2013, PASP, 125, 306

Gaia Collaboration 2018, VizieR Online Data Catalog: I/345

Gaidos, E., Mann, A. W., Lépine, S., et al. 2014, MNRAS, 443, 2561

Gustafsson, B., Edvardsson, B., Eriksson, K., et al. 2008, A\&A, 486, 951

Horne, K. 1986, PASP, 98, 609

Kalas, P., Liu, M. C., \& Matthews, B. C. 2004, Science, 303, 1990

Lagrange, A. M., Kasper, M., Boccaletti, A., et al. 2009, A\&A, 506, 927

Lavail, A., Kochukhov, O., \& Wade, G. A. 2018, MNRAS, 479, 4836

Mamajek, E. E., \& Bell, C. P. M. 2014, MNRAS, 445, 2169

Mann, A. W., Newton, E. R., Rizzuto, A. C., et al. 2016, AJ, 152, 61

Morin, J., Donati, J. F., Petit, P., et al. 2010, MNRAS, 407, 2269

Moutou, C., Dalal, S., Donati, J. F., et al. 2020, A\&A, in press, https://doi . org/10.1051/0004-6361/202038108

Newton, E. R., Mann, A. W., Tofflemire, B. M., et al. 2019, ApJ, 880, L17

Ohta, Y., Taruya, A., \& Suto, Y. 2005, ApJ, 622, 1118

Pepe, F., Mayor, M., Galland, F., et al. 2002, A\&A, 388, 632

Piskunov, N. E., Kupka, F., Ryabchikova, T. A., Weiss, W. W., \& Jeffery, C. S. 1995, A\&AS, 112, 525

Plavchan, P., Barclay, T., Gagné, J., et al. 2020, Nature, 582, 497

Rayner, J., Tokunaga, A., Jaffe, D., et al. 2016, in Society of Photo-Optical Instrumentation Engineers (SPIE) Conference Series, Proc. SPIE, 9908, 990884

Torres, C. A. O., Quast, G. R., da Silva, L., et al. 2006, A\&A, 460, 695

Triaud, A. H. M. J. 2018, The Rossiter-McLaughlin Effect in Exoplanet Research (Switzerland: Springer International Publishing AG), 2

White, R. J., Schaefer, G., Ten Brummelaar, T., et al. 2015, Am. Astron. Soc. Meet. Abstr., 225, 348.12 
E. Martioli et al.: Spin-orbit alignment and magnetic activity in the young planetary system AU Mic

\section{Appendix A: Radial velocity data}

Table A.1. Radial velocity data of AU Mic measured by SPIRou with the mean RV of $-4.3917 \mathrm{~km} \mathrm{~s}^{-1}$ subtracted.

\begin{tabular}{|c|c|}
\hline BJD & $\begin{array}{c}\mathrm{RV} \\
\left(\mathrm{m} \mathrm{s}^{-1}\right)\end{array}$ \\
\hline 2458651.9294631 & $13.4 \pm 13.3$ \\
\hline 2458651.9313410 & $15.0 \pm 12.7$ \\
\hline 2458651.9331425 & $22.9 \pm 9.6$ \\
\hline 2458651.9349485 & $12.6 \pm 10.5$ \\
\hline 2458651.9367580 & $11.1 \pm 7.6$ \\
\hline 2458651.9386249 & $2.7 \pm 7.7$ \\
\hline 2458651.9404333 & $4.2 \pm 7.3$ \\
\hline 2458651.9422373 & $3.6 \pm 8.5$ \\
\hline 2458651.9440437 & $8.4 \pm 8.1$ \\
\hline 2458651.9459171 & $9.3 \pm 6.3$ \\
\hline 2458651.9477209 & $11.1 \pm 6.6$ \\
\hline 2458651.9495294 & $10.4 \pm 8.3$ \\
\hline 2458651.9513325 & $-2.3 \pm 7.6$ \\
\hline 2458651.9532062 & $-5.0 \pm 5.4$ \\
\hline 2458651.9550775 & $-7.0 \pm 5.2$ \\
\hline 2458651.9568821 & $-3.1 \pm 4.8$ \\
\hline 2458651.9586890 & $-4.2 \pm 4.9$ \\
\hline 2458651.9605578 & $2.2 \pm 2.3$ \\
\hline 2458651.9624290 & $0.3 \pm$ \\
\hline 2458651.9642418 & $-3.1 \pm 3.4$ \\
\hline 2458651.9660419 & $-2.6 \pm 3.9$ \\
\hline 2458651.9678465 & $-7.0 \pm 4.8$ \\
\hline 2458651.9697194 & $-1.8 \pm 4.6$ \\
\hline 2458651.9715249 & $-3.3 \pm 2.6$ \\
\hline 2458651.9733311 & $-6.9 \pm 3.6$ \\
\hline 2458651.9751368 & $-14.3 \pm 4.2$ \\
\hline 2458651.9770093 & $-10.4 \pm 4.5$ \\
\hline 2458651.9788159 & $-6.9 \pm 4.2$ \\
\hline 2458651.9806213 & $3.1 \pm 5.1$ \\
\hline 2458651.9824924 & $-3.2 \pm 2.8$ \\
\hline 2458651.9843032 & $-6.7 \pm 4.9$ \\
\hline 2458651.9861111 & $-12.5 \pm 4.6$ \\
\hline 2458651.9879170 & $-11.9 \pm 4.8$ \\
\hline 2458651.9897810 & $-11.2 \pm 3.9$ \\
\hline 2458651.9916527 & $-10.7 \pm 3.3$ \\
\hline 2458651.9934624 & $-2.9 \pm 1.9$ \\
\hline 2458651.9952670 & $-0.7 \pm 2.6$ \\
\hline 2458651.9970739 & $-5.8 \pm 2.7$ \\
\hline 2458651.9989478 & $-9.0 \pm 3.0$ \\
\hline 2458652.0007502 & $-11.5 \pm 3.2$ \\
\hline 2458652.0025548 & $-18.7 \pm 3.8$ \\
\hline 2458652.0044254 & $-2.1 \pm 3.6$ \\
\hline 2458652.0062317 & $-12.9 \pm 4.0$ \\
\hline 2458652.0080383 & $-9.7 \pm 4.3$ \\
\hline 2458652.0098456 & $-22.6 \pm 2.6$ \\
\hline 2458652.0116529 & $-10.5 \pm 3.3$ \\
\hline 2458652.0135269 & $-16.5 \pm 3.9$ \\
\hline 2458652.0153300 & $-14.2 \pm 3.8$ \\
\hline 2458652.0171348 & $-17.7 \pm 2.8$ \\
\hline 2458652.0189428 & $-16.0 \pm 6.0$ \\
\hline 2458652.0208140 & $-23.7 \pm 6.1$ \\
\hline 2458652.0226186 & $-23.0 \pm 4.7$ \\
\hline 2458652.0244869 & $-9.2 \pm 4.3$ \\
\hline 2458652.0263629 & $-6.7 \pm 2.9$ \\
\hline 2458652.0282321 & $-10.0 \pm 4.2$ \\
\hline 2458652.0300386 & $-15.0 \pm 8.3$ \\
\hline
\end{tabular}

Table A.1. continued.

\begin{tabular}{|c|c|}
\hline BJD & $\begin{array}{c}\mathrm{RV} \\
\left(\mathrm{m} \mathrm{s}^{-1}\right)\end{array}$ \\
\hline 2458652.0318420 & $-7.0 \pm 9.7$ \\
\hline 2458652.0336501 & $-0.2 \pm 6.8$ \\
\hline 2458652.0355208 & $6.4 \pm 5.3$ \\
\hline 2458652.0373361 & $-0.6 \pm 6.5$ \\
\hline 2458652.0391326 & $-10.8 \pm 7.9$ \\
\hline 2458652.0409384 & $-15.0 \pm 7.9$ \\
\hline 2458652.0428097 & $-21.7 \pm 4.8$ \\
\hline 2458652.0446176 & $-27.2 \pm 4.5$ \\
\hline 2458652.0464214 & $-25.6 \pm 4.9$ \\
\hline 2458652.0482933 & $-17.3 \pm 5.0$ \\
\hline 2458652.0501654 & $-25.0 \pm 2.5$ \\
\hline 2458652.0519706 & $-27.7 \pm 2.6$ \\
\hline 2458652.0537768 & $-28.9 \pm 2.8$ \\
\hline 2458652.0555851 & $-28.1 \pm 3.4$ \\
\hline 2458652.0574541 & $-19.2 \pm 4.3$ \\
\hline 2458652.0592612 & $-14.4 \pm 4.7$ \\
\hline 2458652.0610671 & $-9.4 \pm 4.9$ \\
\hline 2458652.0629438 & $-11.6 \pm 5.1$ \\
\hline 2458652.0648082 & $-3.9 \pm 5.9$ \\
\hline 2458652.0666136 & $0.1 \pm 3.3$ \\
\hline 2458652.0684188 & $4.2 \pm 3.8$ \\
\hline 2458652.0702908 & $0.7 \pm 4.0$ \\
\hline 2458652.0721621 & $4.6 \pm 3.9$ \\
\hline 2458652.0739677 & $6.7 \pm 5.1$ \\
\hline 2458652.0757738 & $2.7 \pm 5.8$ \\
\hline 2458652.0776455 & $-3.5 \pm 4.3$ \\
\hline 2458652.0795174 & $7.8 \pm 4.4$ \\
\hline 2458652.0813268 & $20.3 \pm 3.5$ \\
\hline 2458652.0831301 & $26.9 \pm 3.9$ \\
\hline 2458652.0849380 & $28.5 \pm 2.2$ \\
\hline 2458652.0868071 & $24.5 \pm 3.4$ \\
\hline 2458652.0886184 & $20.8 \pm 3.7$ \\
\hline 2458652.0904247 & $28.0 \pm 5.0$ \\
\hline 2458652.0922889 & $18.8 \pm 5.4$ \\
\hline 2458652.0941018 & $25.1 \pm 5.7$ \\
\hline 2458652.0959712 & $23.5 \pm 5.5$ \\
\hline 2458652.0977723 & $25.1 \pm 5.5$ \\
\hline 2458652.0996440 & $22.1 \pm 5.0$ \\
\hline 2458652.1015193 & $19.5 \pm 4.6$ \\
\hline 2458652.1033195 & $27.8 \pm 3.0$ \\
\hline 2458652.1051266 & $25.2 \pm 2.8$ \\
\hline 2458652.1069325 & $24.8 \pm 3.7$ \\
\hline 2458652.1088048 & $22.0 \pm 3.2$ \\
\hline 2458652.1106092 & $16.2 \pm 3.2$ \\
\hline 2458652.1124186 & $12.2 \pm 3.2$ \\
\hline 2458652.1142236 & $11.6 \pm 3.3$ \\
\hline 2458652.1160988 & $-0.9 \pm 4.1$ \\
\hline 2458652.1179010 & $1.1 \pm 4.1$ \\
\hline 2458652.1197077 & $3.4 \pm 5.4$ \\
\hline 2458652.1215767 & $13.4 \pm 5.2$ \\
\hline 2458652.1233840 & $12.3 \pm 4.9$ \\
\hline 2458652.1251891 & $12.8 \pm 4.8$ \\
\hline 2458652.1269964 & $9.0 \pm 6.0$ \\
\hline 2458652.1288033 & $5.6 \pm 7.0$ \\
\hline 2458652.1306100 & $1.3 \pm 7.8$ \\
\hline 2458652.1324193 & $9.0 \pm 6.9$ \\
\hline 2458652.1342873 & $6.2 \pm 4.7$ \\
\hline 2458652.1361572 & $11.4 \pm 3.3$ \\
\hline 2458652.1379697 & $7.7 \pm 4.4$ \\
\hline 2458652.1397685 & $10.8 \pm 6.4$ \\
\hline
\end{tabular}


Table A.2. Radial velocity data of AU Mic measured by iSHELL.

\begin{tabular}{lc}
\hline \hline BJD & $\begin{array}{c}\text { RV } \\
\left(\mathrm{m} \mathrm{s}^{-1}\right)\end{array}$ \\
\hline 2458651.9699598 & $19.0 \pm 20.5$ \\
2458651.9715484 & $2.1 \pm 21.9$ \\
2458651.9731365 & $17.4 \pm 27.8$ \\
2458651.9747251 & $31.6 \pm 24.6$ \\
2458651.9763132 & $13.1 \pm 22.6$ \\
2458651.9779024 & $15.8 \pm 23.9$ \\
2458651.9794905 & $23.2 \pm 22.8$ \\
2458651.9810791 & $29.0 \pm 23.2$ \\
2458651.9826683 & $19.1 \pm 23.1$ \\
2458651.9842570 & $5.0 \pm 25.3$ \\
2458651.9858462 & $41.2 \pm 21.2$ \\
2458651.9874342 & $16.8 \pm 19.7$ \\
2458651.9890229 & $13.9 \pm 20.0$ \\
2458651.9906121 & $18.9 \pm 21.3$ \\
2458651.9922002 & $21.3 \pm 18.4$ \\
2458651.9937888 & $16.9 \pm 17.6$ \\
2458651.9953769 & $23.9 \pm 24.3$ \\
2458651.9969661 & $16.5 \pm 23.0$ \\
2458651.9985547 & $23.0 \pm 24.5$ \\
2458652.0001434 & $17.3 \pm 17.6$ \\
2458652.0017320 & $10.8 \pm 23.2$ \\
2458652.0033206 & $6.0 \pm 20.8$ \\
2458652.0049087 & $-1.5 \pm 23.5$ \\
2458652.0064973 & $4.7 \pm 20.0$ \\
2458652.0080860 & $-10.2 \pm 20.1$ \\
2458652.0096740 & $11.0 \pm 19.3$ \\
2458652.0112633 & $19.2 \pm 21.6$ \\
2458652.0128519 & $-6.3 \pm 15.5$ \\
2458652.0144400 & $8.1 \pm 20.7$ \\
2458652.0160280 & $2.6 \pm 26.8$ \\
2458652.0176172 & $1.2 \pm 21.6$ \\
2458652.0192059 & $-28.9 \pm 23.7$ \\
2458652.0207945 & $1.1 \pm 23.7$ \\
2458652.0223837 & $-6.4 \pm 21.1$ \\
2458652.0239724 & $27.6 \pm 24.9$ \\
2458652.0255616 & $-2.9 \pm 16.3$ \\
2458652.0271496 & $32.4 \pm 20.4$ \\
2458652.0287383 & $-2.8 \pm 16.5$ \\
2458652.0303263 & $-22.7 \pm 16.1$ \\
2458652.0319150 & $-10.7 \pm 25.1$ \\
2458652.0335030 & $-11.8 \pm 20.4$ \\
2458652.0350917 & $-3.6 \pm 27.3$ \\
2458652.0366797 & $-10.2 \pm 17.8$ \\
2458652.0382678 & $-8.7 \pm 18.5$ \\
2458652.0398559 & $-12.5 \pm 22.6$ \\
2458652.0414439 & $-6.9 \pm 17.2$ \\
2458652.0430326 & $-13.1 \pm 20.2$ \\
\hline &
\end{tabular}


E. Martioli et al.: Spin-orbit alignment and magnetic activity in the young planetary system AU Mic

\section{Appendix B: Longitudinal magnetic field data}

Table B.1. Longitudinal magnetic field data of AU Mic measured by SPIRou.
Table B.1. continued.

\begin{tabular}{|c|c|}
\hline BJD & $\begin{array}{c}B_{\ell} \\
(\mathrm{G})\end{array}$ \\
\hline 2458652.0320535 & $44.4 \pm 7.7$ \\
\hline 2458652.0338779 & $43.3 \pm 7.7$ \\
\hline 2458652.0357005 & $42.1 \pm 7.5$ \\
\hline 2458652.0375226 & $40.8 \pm 7.5$ \\
\hline 2458652.0393448 & $40.8 \pm 7.4$ \\
\hline 2458652.0411652 & $39.7 \pm 7.5$ \\
\hline 2458652.0429874 & $39.2 \pm 7.5$ \\
\hline 2458652.0448261 & $40.6 \pm 7.6$ \\
\hline 2458652.0466651 & $40.6 \pm 7.5$ \\
\hline 2458652.0485033 & $41.0 \pm 7.5$ \\
\hline 2458652.0503422 & $41.9 \pm 7.6$ \\
\hline 2458652.0521651 & $45.8 \pm 7.6$ \\
\hline 2458652.0539873 & $48.2 \pm 7.8$ \\
\hline 2458652.0558099 & $49.6 \pm 7.9$ \\
\hline 2458652.0576325 & $49.4 \pm 7.9$ \\
\hline 2458652.0594722 & $49.0 \pm 8.0$ \\
\hline 2458652.0613107 & $47.1 \pm 8.1$ \\
\hline 2458652.0631488 & $43.6 \pm 8.1$ \\
\hline 2458652.0649867 & $40.4 \pm 7.9$ \\
\hline 2458652.0668235 & $40.7 \pm 7.8$ \\
\hline 2458652.0686619 & $38.4 \pm 7.8$ \\
\hline 2458652.0705005 & $38.2 \pm 8.0$ \\
\hline 2458652.0723392 & $42.3 \pm 8.4$ \\
\hline 2458652.0741779 & $39.9 \pm 8.6$ \\
\hline 2458652.0760167 & $44.0 \pm 8.7$ \\
\hline 2458652.0778565 & $43.2 \pm 8.6$ \\
\hline 2458652.0796956 & $41.1 \pm 8.5$ \\
\hline 2458652.0815187 & $38.2 \pm 8.5$ \\
\hline 2458652.0833411 & $37.4 \pm 8.4$ \\
\hline 2458652.0851640 & $38.2 \pm 8.5$ \\
\hline 2458652.0869876 & $37.8 \pm 8.5$ \\
\hline 2458652.0888254 & $38.1 \pm 8.5$ \\
\hline 2458652.0906491 & $40.1 \pm 8.4$ \\
\hline 2458652.0924873 & $40.9 \pm 8.2$ \\
\hline 2458652.0943242 & $40.1 \pm 8.1$ \\
\hline 2458652.0961630 & $37.9 \pm 7.9$ \\
\hline 2458652.0980173 & $37.2 \pm 7.8$ \\
\hline 2458652.0998544 & $38.1 \pm 7.9$ \\
\hline 2458652.1016930 & $40.0 \pm 8.0$ \\
\hline 2458652.1035151 & $39.6 \pm 8.0$ \\
\hline 2458652.1053364 & $40.5 \pm 8.0$ \\
\hline 2458652.1071589 & $42.8 \pm 7.9$ \\
\hline 2458652.1089819 & $44.6 \pm 7.8$ \\
\hline 2458652.1108047 & $48.9 \pm 7.8$ \\
\hline 2458652.1126282 & $50.0 \pm 7.8$ \\
\hline 2458652.1144511 & $52.8 \pm 7.7$ \\
\hline 2458652.1162734 & $51.9 \pm 7.6$ \\
\hline 2458652.1181116 & $51.0 \pm 7.6$ \\
\hline 2458652.1199329 & $49.0 \pm 7.5$ \\
\hline 2458652.1217550 & $47.2 \pm 7.5$ \\
\hline 2458652.1235772 & $44.2 \pm 7.5$ \\
\hline 2458652.1253838 & $41.4 \pm 7.5$ \\
\hline 2458652.1271903 & $39.2 \pm 7.5$ \\
\hline 2458652.1289979 & $34.9 \pm 7.5$ \\
\hline 2458652.1308206 & $34.2 \pm 7.5$ \\
\hline 2458652.1326591 & $32.7 \pm 7.6$ \\
\hline 2458652.1344990 & $33.3 \pm 7.6$ \\
\hline 2458652.1363363 & $30.6 \pm 7.7$ \\
\hline
\end{tabular}




\section{Appendix C: Posterior distributions}

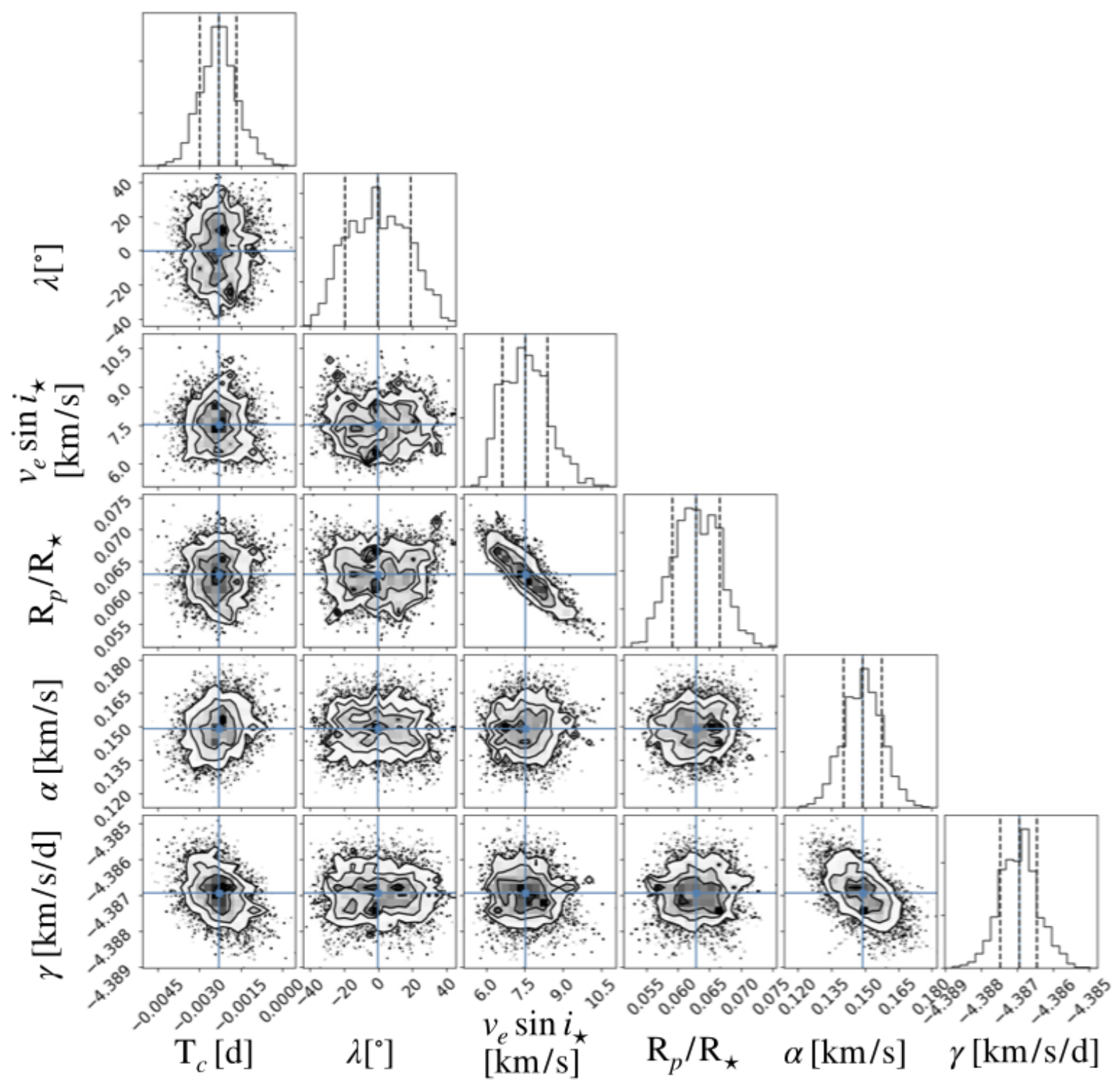

Fig. C.1. Pairs plot showing the MCMC samples and posterior distributions for the six free parameters presented in Table 2. The contours mark the $1 \sigma, 2 \sigma$, and $3 \sigma$ regions of the distribution. The gray scale shades illustrate the density of samples, where darker means denser. The blue crosses indicate the best fit values for each parameter and the dashed vertical lines in the projected distributions indicate the median value and the $1 \sigma$ uncertainty ( $34 \%$ on each side of the median). 
E. Martioli et al.: Spin-orbit alignment and magnetic activity in the young planetary system AU Mic

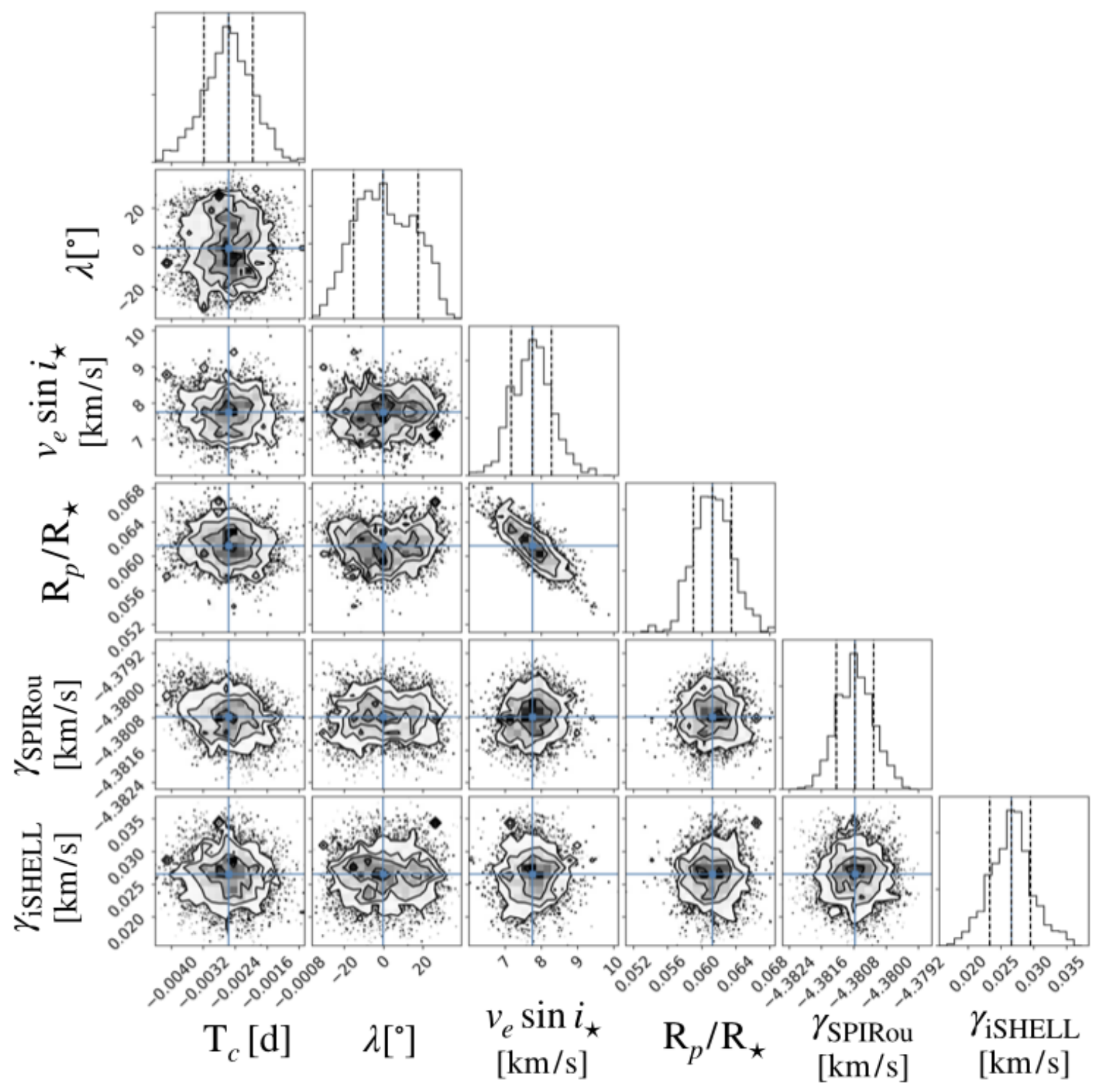

Fig. C.2. Pairs plot showing the MCMC samples and posterior distributions for the six free parameters presented in Table 3. The contours mark the $1 \sigma, 2 \sigma$, and $3 \sigma$ regions of the distribution. The gray scale shades illustrate the density of samples, where darker means denser. The blue crosses indicate the best fit values for each parameter and the dashed vertical lines in the projected distributions indicate the median value and the $1 \sigma$ uncertainty (34\% on each side of the median). 